\title{
Reply to the Letter to the Editor by Reza Pakzad and Saeid Safiri, integration of MRI to clinical nomogram for predicting pathological stage before radical prostatectomy: bias in the prediction model
}

\author{
Cedric Lebacle $^{1}{ }^{1} \cdot$ Laurent Salomon $^{1} \cdot$ Alexandre De La Taille $^{1}$. \\ Françoise Roudot-Thoraval ${ }^{2}$
}

Received: 28 September 2017 / Accepted: 5 October 2017 / Published online: 17 October 2017

(c) Springer-Verlag GmbH Germany 2017

Dear Editor,

Reza Pakzad and Saeid Safiri have suggested a methodological point about our publication "Integration of MRI to clinical nomogram for predicting pathological stage before radical prostatectomy" published in World Journal of Urology [1].

They suggest that the multivariate findings may be misleading due to a testimation bias in model building [2]. In fact, explanatory variables with almost small effect would not be entered into the multivariate model and explanatory variables with almost large effect would be only entered into the final multivariate model when the variables is selected to be included in the multivariate model based on the $p \leq 0.05$. The relax $p$ values including ( $p$ value $\leq 0.1$ or $p$ value $\leq 0.2$ ) is suggested to be considered for the variable selection of multivariate models to decrease the aforementioned bias.

This is a pertinent suggestion. In fact, in our study, all the variables with a $p$ value $\leq 0.20$ have been included in the model building. We have included MRI $(p<0.001)$, clinical stage $(p<0.001)$, serum PSA level $(p=0.001)$, biopsy

This reply refers to the article available at doi:10.1007/s00345017-2020-x.

Electronic supplementary material The online version of this article (doi:10.1007/s00345-017-2100-y) contains supplementary material, which is available to authorized users.

Cedric Lebacle

cedric.lebacle@gmail.com

1 Department of Urology, CHU Mondor, Assistance Publique des Hopitaux de Paris, University Paris-Est, Creteil, France

2 Department of Public Health, CHU Mondor, Assistance Publique des Hopitaux de Paris, University Paris-Est, Creteil, France
Gleason score $(p<0.001)$ and prostate weight $(p=0.013)$. The variable we have not included was the age $(<$ or $>60)$ with a $p=0.527$ in univariate analysis (Table S1). There were, therefore, no variables with a $p$ value $\leq 0.20$ in univariate not included in the multivariate model.

Furthermore, the testimation bias is important for small sample. In our large sample of 1743 patients (1054 patients in test-group and 689 in the validation group). The testimation bias is much smaller in substantial sample sizes [2].

Authors contributions CL and LS: Project development, data collection and management, data analysis, manuscript writing and editing. FR-T: Data analysis, manuscript writing and editing. ADe LaT: Data collection and management, manuscript writing and editing.

\section{Compliance with ethical standards}

Informed consent For this type of study formal consent is not required.

Conflict of interest Authors of this article have no conflict of interest to declare.

\section{References}

1. Lebacle C, Roudot-Thoraval F, Moktefi A, Bouanane M, De La Taille A, Salomon L (2016) Integration of MRI to clinical nomogram for predicting pathological stage before radical prostatectomy. World J Urol. doi:10.1007/s00345-016-1981-5

2. Steyerberg E (2008) Clinical prediction models-a practical approach to development, validation, and updating. Springer, Berlin 